\title{
GEOLOGÍA Y ESTRATIGRAFÍA DE LA HOJA ABANGARES GUANACASTE, COSTA RICA
}

\author{
Kennet Flores ${ }^{1}$, Percy Denyer ${ }^{2} \&$ Teresita Aguilar ${ }^{2}$ \\ ${ }^{1}$ Institut de Géologie et Paléontologie, Université de Lausanne, BFSH2, CH-1015 \\ Lausanne, Suiza \\ Kennet.FloresReyes@igp.inil.ch \\ ${ }^{2}$ Escuela Centroamericana de Geología, Universidad de Costa Rica
}

\section{INTRODUCCIÓN}

En este trabajo se resumen los resultados propuestos por Flores (2003) en cuanto al reordenamiento litoestratigráfico y las investigaciones realizadas por los autores en el marco del proyecto de la Vicerrectoría de Investigación \# 113-90-071, Análisis Geoestructural Comparado de Costa Rica.

Se hacen algunas modificaciones en cuanto al concepto estratigráfico y cartografiado presentado por Rivier (1983) para la hoja Abangares (Fig. 1). Se definen tres unidades nuevas: Formación Coyolito, Formación San Buenaventura (Flores, 2003) y el Miembro Loma Danta de la Formación Descartes. Así como la redefinición de las formaciones Barbudal y El Viejo y el Miembro Cerco de Piedra, de la Formación Descartes (Flores, 2003) (Fig. 2).

La base de la secuencia sedimentaria en el área de estudio esta compuesta por las secuencias epiclásticas, volcaniclásticas y bituminosas de origen pelágico, hemipelágico y turbidítico del Cenomaniano Superior al Turoniano Inferior, que componen la Formación Berrugate (Flores et al., 2003).
La Fm. Sabana Grande esta compuesta por sedimentos silíceos y carbonatados de origen pélagico, hemipelágico y turbidítico, cuya edad, probablemente corresponde con el Cenomaniamo- Coniaciano. Esto excluye a los sedimentos turbidíticos y hemipelágicos, del Coniaciano al Maastrichtiano Inferior basal, que ahora corresponderían

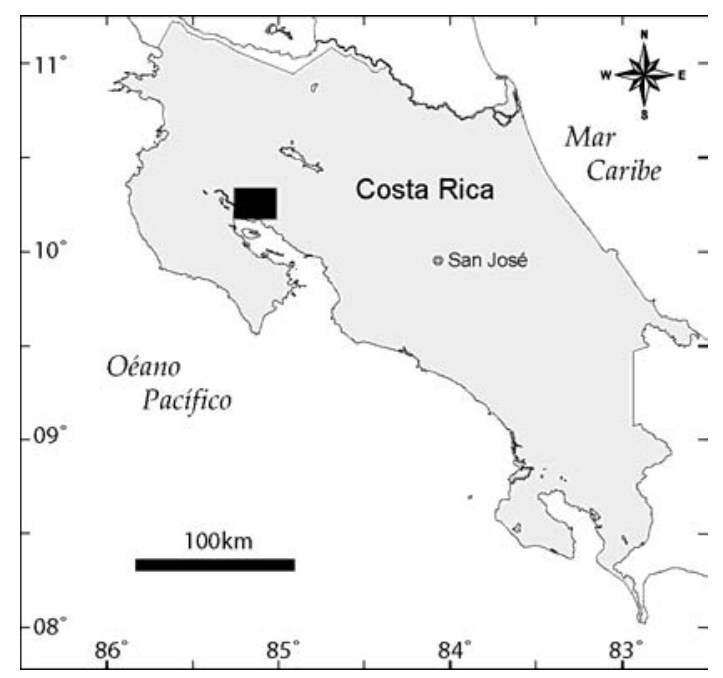

Fig. 1: Mapa de ubicación del área de estudio 


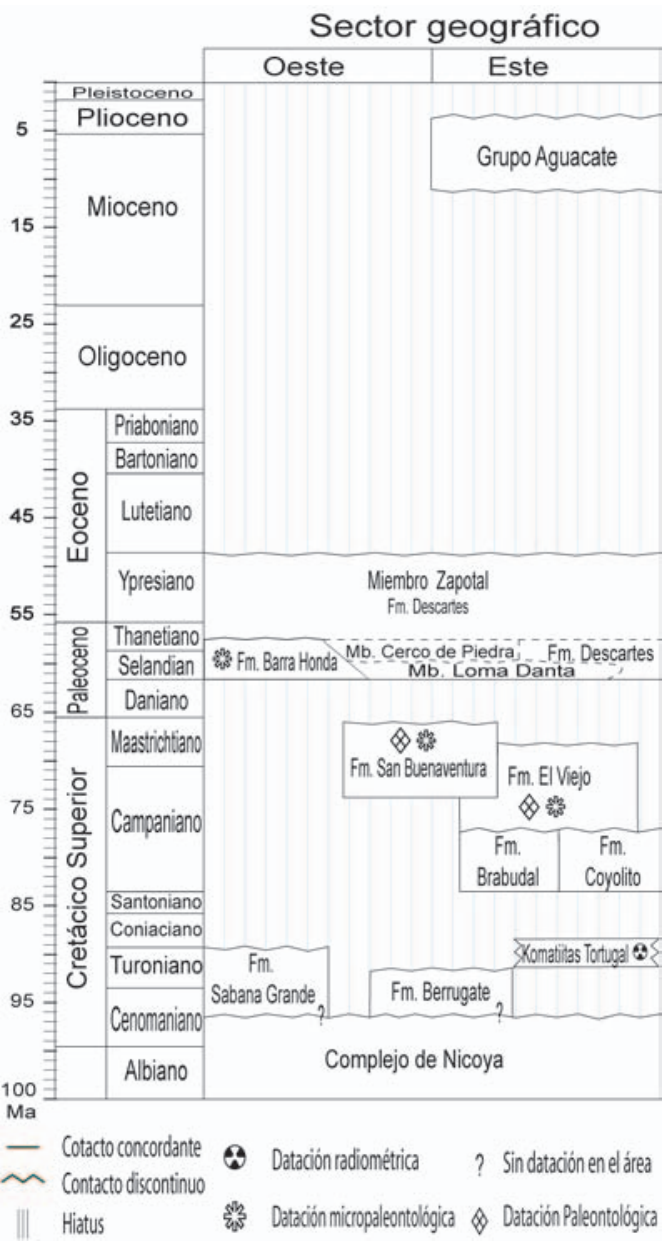

Fig. 2: Columna cronoestratigráfica. Escala de tiempo basada en ICS.

con las formaciones Nambí y Piedras Blancas (Flores et al., 2003).

La Formación Barbudal se restringe a las secuencias sedimentarias del Campaniano Inferior compuestas por conglomerados con clastos de basaltos y algunos de komatitas(?) aflorantes en el área este del bajo Tempisque (Flores, 2003), por lo que se propone separar de esta unidad los sedimentos calcáreos, anteriormente conocidos como unidad Colorado (Campañas Geológicas de 1976 y 1977), ya que estos no presentan asociación alguna con los sedimentos típicos de esta unidad, descritos por Rivier (1983). Se propone que solo se use el nombre de Formación Barbudal, pues el término Formación Conglomerado Barbudal, restringe litológicamente a la unidad, lo cual imposibilitaría su correlación litológica y estratigráfica con otras unidades (Flores, 2003).

En el área de estudio, la Formación El Viejo corresponde con los sedimentos turbidíticos carbonatados del Campaniano Inferior terminal al Maastrichtiano Inferior (Flores, 2003), que afloran en el área de Colorado de Abangares y cerro Congo. Para esta unidad se propone abandonar el término de Formación Calizas El Viejo (Galli-Oliver $\&$ Schmidt-Effing, 1977), pues aunque las calizas son la litología característica de esta unidad, no son el único tipo de roca incluida en esta formación. Por el contrario, solo representan el $25 \%$ de las rocas que la componen. Además, este nombre evidencia un concepto erróneo del origen de la mayoría de las rocas de esta unidad, pues el término de caliza es usualmente asociado con plataformas carbonatadas típicas y esta unidad es de origen turbidítico (Flores, 2003).

\section{COMPLEJO DE NICOYA}

Esta unidad no se detalla en el presente trabajo; sin embargo, se hacen algunas consideraciones generales, con base en Dengo (1962) y Kuijpers (1980). Corresponde con una secuencia ofiolítica basáltica más antigua que el Campaniano-Santoniano, compuesta por basaltos toleíticos, que ocurren en forma masiva y en almohadillas, diques, brechas de hialoclastita. Otras rocas asociadas son radiolaritas y rocas intrusitas como gabros, ferrogabros, diabasas, picritas y plagiogranitos.

\section{KOMATIITAS TORTUGAL}

Descripción: Rocas volcánicas ultramáficas de alto magnesio y fenocristales de olivino.

Estratotipo: Los afloramientos de Tortugal (426,5/239,2 hoja Juntas). Los afloramientos de Pueblo Nuevo $(414,4 / 245,7)$ y el tajo Higuerillas (422,3/240,7), ambos sitios en la hoja Abangares, pueden servir de paraestratotipo. 
Aspectos regionales: Tienen similitud petrológica y de edad con afloramientos de Gorgona y $\mathrm{Cu}$ razao (Alvarado et al., 1997).

Edad geológica: 89,7 \pm 1,4 Ma (Alvarado et al. (1997), la cual corresponde con el Turoniano al Coniaciano Inferior.

Relaciones estratigráficas: Son inciertas, se presume que parte de las rocas del Complejo de Nicoya son más antiguas. La komatiita está intruída por diques de basalto, cuyo espesor varía de $2 \mathrm{~cm}$ a varios metros.

Aspectos específicos: Aflora en cerros de pocos metros de altura (menos de $15 \mathrm{~m}$ ). El patrón de afloramientos general tiene una forma elongada N60W, de $14 \mathrm{~km}$ de longitud y 1,5 km de ancho. $\mathrm{Su}$ forma semeja un dique.

Antecedentes: Aguilar (1977) reportó un basalto con más de $50 \%$ olivino modal en Pueblo Nuevo de Colorado. Galli (1979) interpretó esta roca como una harzburgita. Tournon (1994) indica la presencia de un bloque de picrita en la misma área. Calero (1987) describió estas rocas como lamprófidos. Alvarado et al. (1997) y Alvarado \& Denyer (1997) las describen como komatiitas y presentan un mapa geológico.

\section{FORMACIÓN COYOLITO}

Descripción: La parte inferior está compuesta por secuencias de lutitas rojizas y violetas con intercalaciones milimétricas y centimétricas de areniscas finas gris verdosas. Seguido por secuencias de intercalaciones centimétricas, cíclicas de lutitas violetas y areniscas finas gris verdosas, ambas predominantemente silíceas. La parte superior está caracterizada por la presencia de estratos decimétricos de areniscas medias a gruesas, gris verdosas, predominantemente de composición volcánica.

Estratotipo: Se propone como localidad tipo los afloramientos en un tajo al sur del cerro Piedras
Negras $(237,8 \mathrm{~N} / 427,6 \mathrm{E}$, hoja Chapernal). En esta localidad se presenta la unidad inferior con aproximadamente $50 \mathrm{~m}$ y está altamente plegado. Seguido por $15 \mathrm{~m}$ de intercalaciones de areniscas gris calcáreas decimétricas y lutitas violetas centimétricas, $5 \mathrm{~m}$ de intercalaciones centimétricas de lutitas violetas y areniscas grises calcáreas. La secuencia culmina con un nivel de $2 \mathrm{~m}$ de intercalaciones decimétricas de areniscas gruesas verdes. Como localidades auxiliares se propone los afloramientos junto a la carretera entre Coyolito y la Peña (hoja Chapernal).

Aspectos regionales: El espesor máximo estimado es de $200 \mathrm{~m}$ en el área del cerro Piedras Negras. Esta unidad aflora en las hojas Abangares, Berrugate, Juntas y Chapernal (escala 1:50 000, IGN). La secuencia se presenta con estratificación milimétrica a centimétrica mayoritariamente. Los estratos son planos continuos, de geometría tabular, se presentan moderadamente a intensamente plegados, producto de deformación sinsedimentaria y tectónica. No se descarta que en algunas localidades, las secuencias puedan presentar repeticiones debido a fallas inversas de muy bajo ángulo.

Relaciones estratigráficas: Sobreyace inconformemente a los basaltos del Complejo de Nicoya y disconformemente a los sedimentos de la Formación Berrugate. Esta sobreyacida discordantemente por las formaciones El Viejo y Descartes, ambos contactos son erosivos (Aguilar \& Denyer, 2002).

Edad geológica: Basados en la posición estratigráfica se propone una edad del Campaniano Inferior.

Aspectos específicos: Las rocas de esta unidad son comúnmente usadas como material de préstamo, para los caminos vecinales.

Antecedentes: Fue descrita como unidad informal Coyolito en la Campaña geológica de 1987. Denyer et al. (1987) incluyen la unidad Coyolito dentro de la Formación Rivas. Aguilar \& Denyer (2002) utilizan el término Unidad Coyolito. 


\section{FORMACIÓN SAN BUENAVENTURA}

Corresponde con una secuencia de sedimentos clásticos de origen turbidítico, que aflora en el sector de Colorado de Abangares. Esta unidad se caracteriza por presentar un alto contenido fosilífero.

Descripción: Está compuesta por unos 400 m de intercalaciones decimétricas a centimétricas de areniscas finas a medias gris verdosa y lutitas grises a negras calcáreas. Se presentan medianamente deformadas, con pliegues de corta amplitud, cerrados y discontinuos, lo que evidencia una deformación sinsedimentaria. Dentro de algunos estratos de lutitas gris verdosas se determinaron fósiles, tales como fragmentos y especímenes completos de amonites, escafópodos, bivalvos nacarados, gasterópodos y fragmentos de moluscos $(243,75 \mathrm{~N} / 413,2 \mathrm{E}$ y $242,05 \mathrm{~N} / 410,40 \mathrm{E})$. Microscópicamente las areniscas finas corresponden con wackes arcósicas. También se observaron horizontes de $2 \mathrm{~m}$ de espesor, compuestos por estratos decimétricos a centimétricos de areniscas medias negras calcáreas y calizas arenosas con fragmentos de rudistas y gasterópodos que microscópicamente corresponden con packstones con fragmentos de rudistas.

Estratotipo: Como localidad tipo se propone el afloramiento entre la parte alta del tajo los Almendros y el cerro Cerco de Piedra (243,7N/ 413,85E a $243,65 / 413,2 E)$. En esta localidad aflora una secuencia de $400 \mathrm{~m}$ compuesta por intercalaciones centimétricas de areniscas finas y lutitas gris verdosas calcáreas y al menos un horizonte de $2 \mathrm{~m}$ de areniscas medias calcáreas y calizas arenosas negras. Como localidades auxiliares se proponen las quebradas San Buenaventura y Pilas, en el área de Colorado de Abangares.

Aspectos regionales: En el área de la localidad tipo se observó un espesor total de $400 \mathrm{~m}$. Esta unidad solo aflora en la hoja Abangares y los únicos afloramientos se encontraron en la falda este y sur de los cerros Cerco de Piedra y las quebradas San Buenaventura y Pilas. La secuencia se presenta con estratificación decimétrica a centimétrica, los estratos son planos continuos, de geometría tabular, se presentan leve a intensamente plegados, producto de deformación sinsedimentaria.

Relaciones estratigráficas: Sobreyace concordantemente los sedimentos de la Formación El Viejo, aunque este contacto no se observó claramente, se basa en la similitud litológica entre la parte superior de la Formación El Viejo y los sedimentos de esta unidad; no se descarta que el contacto sea transicional. Está sobreyacida por medio de una discordancia angular erosiva por el Miembro Loma Danta de la Formación Descartes. Según la caracterización petrográfica, la posición estratigráfica, el contenido fosilífero y la edad, esta unidad correspondería al equivalente turbidítico de los sedimentos hemipelágicos de la Formación Piedras Blancas (Flores 2003, Flores et al., 2003).

Edad geológica: Con base en el hallazgo de amonites, de los cuales posiblemente algunos pertenecen al género Pseudokossmaticeras del Campaniano (Schmitdt-Effing, 1974), así como la microfauna de los afloramientos de la quebrada Pilas, reportada por Calvo (1998), se propone una edad Campaniano Superior terminal a Maastrichtiano Superior basal

Aspectos específicos: Schmitdt-Effing (1974) reporta el primer hallazgo de fragmentos de amonites, probablemente del género Pseudokossmaticeras del Campaniano. Calvo (1998) determinó en lutitas, areniscas y calcarenitas de la quebrada Pilas las siguiente microfauna, macroforaminíferos: Sulcoperculina vermunti (Thiadens), Sulcoperculina globosa de Cizancourt, Orbitocyclina minima (H. Douvillé), Sulcorbitoides pardoi Brönnimann, Pseudorbitoides israelsky Vaughan $\&$ Cole, Vaughanina sp., Placopsilina sp., Acervulina sp., Bolivina f. incrassata Reuss, Marsonella cf. oxycona (Reuss), Coskilinoides sp., Praealvolina sp., Vidalina cf. hispanica Schlumberger, Textulariina aglutinados, Miliólidos. Foraminíferos planctónicos: Gansserina gansseri (Bolli), Globotruncanita stuarti (de Lapparent), Globotruncanita stuartiformis (Dalbiez), Globotruncanita sp., Globotruncana bulloides Vogler, 
Globotruncana ventricosa White, Globotrunca$n a$ gr. lapparenti Brotzen-linneiana (d'Orbigny), Globotruncana aegyptiaca Nakkady, Globotruncana arca (Cushman), Globotruncanaella sp. cf. citae (Bolli), Globigerinelloides sp., Rosita sp., Rosita fornicata (Plummer), Rugoglobigerina sp., Pseudoguembelina sp., Pseudotextularia elegans (Rzehnk), Hedbergella sp. Flora: Jania sp., Archaeolithothamniun sp., Lithothamnium sp., Polystrata alba (Pfender) y fragmentos de rudistas, braquiópodos, Inoceramus, Microcodium, serpulidos y espinas de erizos.

Antecedentes: Schmidt-Effing (1974) reporta el primer hallazgo de amonites de América Central meridional en rocas que en este trabajo se asocian a esta unidad. Fue descrita como unidad informal San Buenaventura durante las Campañas Geológicas del 1976 (Adrian Salazar, José Francisco Rojas, Hugo Rodriguez, Jorge Chaves, Jorge Laguna, Julio Elizondo, Jorge Vargas y Aida Santana), 1977 (Teresita Aguilar, Percy Denyer, Jorge Flores, Emilio Garro, Gerardo Jager, Carlos León, Jose Fabio Morera, Franz Ulloa y Rolando Valdez) y 1978 (Francisco Alvarez, Teresa Arrieta, German Leandro, Guillermo Lezama, Sergio Mora, Hannia Azuola, Johny Piedra y Alfonso Sanabria). Campo II (2001) definen por primera vez el Miembro San Buenaventura, dentro del cual incluyen rocas que en este trabajo se correlacionan con la Formación El Viejo y la Formación San Buenaventura.

\section{FORMACIÓN BARRA HONDA}

Esta unidad corresponde con la secuencia carbonatada ampliamente extendida en los márgenes de la desembocadura del río Tempisque. Conforma grandes estructuras geomorfológicas y geológicas de gran belleza, tales como los sistemas cársticos de los parques nacionales Barra Honda y Palo Verde (Flores, 2003).

Descripción: Dengo (1962) la divide en dos partes, la parte inferior compuesta por calizas pobremente estratificadas, duras, con textura homogénea fina y color blanco amarillento. La parte superior, compuesta por calizas bien estratificadas en capas de espesor mediano a delgado, también con textura homogénea fina y color blanco amarillento. Mora (1981) distingue dos subunidades, una primer subunidad estratificada bien expuesta en la zona de lapiaz del Mirador, en el Mogote del cerro Barra Honda, en Fierro, Corralillo y el cerro Panamá. Según la clasificación de Folk, consiste de una biomicrita con variaciones locales a bioesparita y a biopelmicrita. Según la clasificación de Dunham se trata de un packstone con variaciones locales a grainstone y packstone esparítico. Se compone de fragmentos de erizo, algas (Corallinacea), esponjas, bryozoos, radiolarios (Spumellaria) y foraminíferos (Quinqueloculina, Triloculina, y otros). La segunda subunidad es maciza y se observa en la pared sur del cerro Barra Honda y en las paredes este y oeste del cerro Corralillo. Según la clasificación de Folk está formada por una biolitita algal con áreas restringidas de biopelmicrita. Según la clasificación de Dunham es un boundstone en donde la actividad algal ha ligado los demás componentes de la roca. Está cementada en su mayor parte por micrita primaria y por productos de la actividad algal. Jaccard et al. (2001) mencionan que esta unidad también presenta facies que evidencia una erosión de rocas cretácicas (Loma Pochote 236,6N/395,6E, hoja Matambú), pero que contiene foraminíferos típicos del Paleoceno Superior, tal como la Morozovella velascoensis.

Estratotipo: Dengo (1962) propone como localidad tipo los cerros de Barra Honda y como sección típica la que se encuentra en el camino entre Barra Honda y las cumbres de los cerros del mismo nombre.

Aspectos regionales: Dengo (1962) menciona un espesor de $100 \mathrm{~m}$ para la parte inferior y 250 $\mathrm{m}$ para la parte superior. Aflora en las hojas Matambú, Talolinga y Abangares; además, se conoce la existencia de esta unidad en las hojas Matapalo (Cabo Velas), Tempisque y Cañas. La secuencia se presentan con estratificación centimétrica a métrica o bien masiva, los estratos son planos continuos, de geometría tabular. 
Relaciones estratigráficas: Sobreyace discordantemente a los sedimentos de la Formación Curú. Jaccard \& Münster (2000) mencionan que sobreyace discordantemente a una secuencia de sedimentos pelágicos y hemipelágicos asociados a la Formación Sabana Grande en el área de Alto Tempisque. También sobreyace tectónicamente a la Formación Sabana Grande y al Miembro Zapotal, Formación Descartes, por medio de las fallas inversas Calera y Naranjo respectivamente (hoja Matambú). En el área de los fila Catalina, esta sobreyacida por el Miembro Zapotal de la Formación Descartes.

Edad geológica: Jaccard et al. (2000) basados en asociaciones de microfósiles, le asignan una edad del Paleoceno Superior al Eoceno Inferior, la cual posteriormente se restringe a Paleoceno Superior basado en análisis isotópicos de ${ }^{87} \mathrm{Sr} /{ }^{86} \mathrm{Sr}$, de $\delta{ }^{13} \mathrm{C}$ y $\delta{ }^{18} \mathrm{O}$ (Jaccard \& Münster, 2001).

Aspectos específicos: Dengo (1962) menciona la siguiente microfauna determinada por Thalmann, parte inferior: Idalina sp., Quinqueloculina sp., Biloculina (Pyrgo) sp., Dasycladacea (algae), Tricladus sp. cf. T. pinarensis (Keys), Broeckelle sp. cf. B. belgica Morell, Cymopolia sp. cf. elongata (Defr.), Terquemella sp., ? Neomeris sp., Textularia sp., Bigenerina sp., Mesophyllum sp., Solenopora sp., Milliolidae, Lithothamnium y Haplophragmoides sp. Parte superior Astrocoenia sp., Bathysiphon sp., Miliolidae, Dasycladacea (algae), Solenopora sp., Parachaetetes sp., Mesphyllum sp., Miliolidae (Triloculine y Quinqueloculine) y Lithothamniun. Calvo (1987) habla de tres sistemas depositacionales en la Formación Barra Honda, un sistema de plataforma abierta, un sistema de plataforma interna y un sistemas de plataforma retrogradacional. Jaccard et al. (2001) mencionan que todas las facies de la Formación Barra Honda se caracterizan por la presencia de algas rojas (coralinaceas) y de Ethelia alba, las facies micríticas contienen foraminíferos Rotalidos entre las cuales se destacan Thalmannita madrugaensis, Miliólidos y Alveolinidos. En la zona de Palo Verde determinan macroforaminíferos tales como Ranicothalia catenula, Neodiscocyclina barkeri, los cuales están asociados a Ethelia alba. En loma Pochote se determinó la presencia de Morozovella velascoensis y Pentacrinites sp. (crinoideo). Aguilar \& Denyer (2001) reportan el hallazgo de una nueva especie de coral scleractinia en las cercanias de puerto Níspero (hoja Abangares). Esta especie corresponde con Euphyllia donatoi.

Antecedentes: Descrita originalmente por Romanes (1912) como parte de lo que él denominó limestone and cherts. MacDonald (1920) las llamo Calizas de Nicoya y las incluye dentro de lo que el denominó Serie de Nicoya. Jaccard et al. (2001) incluyen dentro de la Formación Barra Honda lo que Calvo \& Bolz (1991) definieron como Formación Espíritu Santo, para las calizas del área de alto Tempisque.

\section{FORMACIÓN DESCARTES}

Esta compuesta por secuencias de sedimentos turbidíticos volcanoclásticos y carbonatados definidos por Astorga (1987). Tienen una amplia distribución geográfica, que comprende desde fila Pajaros, la isla San Lucas, el sureste de la península de Nicoya hasta la península de Santa Elena, bahía Bolaños y el suroeste de Nicaragua. En el área de estudio, se puede dividir en tres miembros, Loma Danta, Cerco de Piedra y Zapotal.

\section{MIEMBRO LOMA DANTA}

Corresponde con un horizonte de sedimentos calcáreos de origen turbidítico, que constituyen la base de la Formación Descartes en el área de los cerros Cerco de Piedra y Congo.

Descripción: En el área de cerro Congo la base está compuesta por $10 \mathrm{~m}$ de areniscas medias gris verdosas calcáreas, mientras que en Colorado de Abangares la base corresponde con una secuencia de 6-10 m, de intercalaciones centimétricas de calcilutitas grises parcialmente silicificadas, que microscópicamente son barros de globigerinas y 
radiolarios recristalizados a calcita. Seguido por una secuencia de $2-5 \mathrm{~m}$ de calizas masivas con lentes de calcarenitas, caracterizados por presentar fragmentos de corales, rudistas, Inoceramus y bivalvos. Microscópicamente, las calizas masivas corresponden con wackestones con algas rojas Ethelia alba, peloides, fragmentos de coral y clastos de micrita retrabajados. Continuan 5-10 $\mathrm{m}$ de calizas arenosas con fragmentos de rudistas, Inoceranus, bivalvos y clastos angulares de lutitas silíceas, en algunas localidades presentan lentes de calcarenitas, que corresponden con packstones con fragmentos de rudistas, algas rojas, Polystrata alba, oncolitos, fragmentos de equinodermos, foraminíferos globosos posibles Discocyclina?, peloides y estilolitos.

Estratotipo: Se propone como localidad tipo dos afloramientos, ambos cercanos al pueblo de San Buenaventura de Colorado (241,4N/410,25E y 241,4N/409,5E). En los que afloran calcilutitas grises, calizas masivas fosilíferas con lentes arenosos o de calcarenitas y calizas arenosas fosilíferas intercaladas.

Aspectos regionales: Se calcula un espesor máximo de $30 \mathrm{~m}$, aunque por lo general los afloramientos no presentan espesores constantes. Aflora en las hojas Abangares y Juntas. Su ubicación se restringe al flanco este y sur de los cerros Cerco de Piedra y el sur del cerro Cajón. La secuencia se presenta masiva o con estratificación centimétrica a decimétrica, los estratos son continuos y de geometría tabular. Las calcarenitas se presentan en estratos discontinuos y de geometría lenticular. Por lo general los afloramientos son de regular calidad y muchos se restringen a bloques y/o relictos sobre la cima de cerros y lomas.

Relaciones Estratigráficas: Sobreyace disconformemente a la Formación San Buenaventura mediante un contacto erosivo. Esta sobreyacida disconformemente por el Miembro Cerco de Piedra de la Formación Descartes, así como por las secuencias turbidíticas de la Formación Descartes en el área de cerro Cajón, por medio de un contacto erosivo en ambas áreas. Esta unidad por su composición y posición estratigráfica, se puede correlacionar con la base de la Formación Barra Honda descrita por Jaccard et al. (2001) en el área de Loma Pochote.

Edad geológica: Aunque no se cuenta con dataciones para esta unidad, con base en correlaciones estratigráficas, así como en su contenido fosilífero, se le asigna una edad de Paleoceno Superior.

Aspectos específicos: Aguilar (1977) reporta la presencia de algas coralinas (Lithothamnium sp.), fragmentos de valvas de moluscos, equinodermos y esponjas calcáreas en las calizas de loma Monte Potrero. Además, en las calizas se identificaron corales solitarios, algas y gasterópodos, en sección delgada se observan alga verdes (Dycladáceas), bryozoos, foraminíferos bentónicos y planctónicos y ostrácodos.

Antecedentes: Fue descrita como unidad informal Loma Danta en las Campañas geológicas de 1976, 1977 y 1978. Sprechmann (1982) se refiere a estas rocas, como litozona informal Loma Danta (calizas alodápicas).

\section{MIEMBRO CERCO DE PIEDRA}

Corresponde con sedimentos arenosos y conglomerádicos de origen turbidítico que afloran en el área de Colorado de Abangares y la isla de Chira.

Descripción: Esta unidad corresponde con conglomerados de fragmentos volcánicos, regular a mal seleccionados, con fragmentos angulares a redondeados de hasta $30 \mathrm{~cm}$, predominantemente andesíticos, de calizas, y de pedernales. Presentan matriz arcillosa a limo arenosa con cemento calcáreo (Sprechmann, 1982). Microscópicamente la matriz corresponde con wackes líticas. Areniscas conglomerádicas de mal a moderadamente seleccionadas, redondeadas a subredondeadas, con cemento calcáreo son de color gris a gris verdoso (Sprechmann, 1982). Microscópicamente corresponden con wackes arcósicas con clastos 
angulares de basaltos y clastos angulares de lodolitas micríticas y wackstone con foraminíferos. Areniscas compactas, mal seleccionadas con componentes angulares a subredondeados de composicíón volcánica (Sprechmann, 1982). Rivier (1983) describe tobas andesíticas de color pardo amarillento a verde, con fragmentos líticos y feldespatos subredondeados, que parecen ser retrabajados. Además, menciona que aparecen andesitas en mantos intercalados y en bloques en los conglomerados, estas son hipocristalinas, gris oscuras, con textura afanítica porfirítica, presentan localmente estructuras fluídas. En localidades del cerro Cerco Piedra $(242,75$ N/410,35E, hoja Abangares) y la isla Paloma (241,7N/339,8E, hoja Berrugate), se encontraron bloques métricos de andesitas con fenocristales de hornblenda, de hasta $3 \mathrm{~cm} \mathrm{x1} \mathrm{cm} \mathrm{(Flores,} \mathrm{2003).}$

Estratotipo: La localidad tipo fue definida por Sprechmann (1982) en el cerro del mismo nombre, localizado en Colorado de Abangares. Como localidades auxiliares se propone la isla Paloma (241,7N/339,8E, hoja Berrugate).

Aspectos regionales: Sprechmann (1982) y Rivier (1983) coinciden en que el espesor máximo es de $500 \mathrm{~m}$. Aflora en las hojas Abangares y Berrugate (escala 1: 50 000, IGN). Sus afloramientos se reducen a los cerros Cerco de Piedra y Zapote en la hoja Abangares, isla Paloma, bajo Negro, bajo Montero y punta Flor en la isla Chira, en la hoja Berrugate. La sección se presenta mal estratificada, es de geometría tabular, con una estratificación subhorizontal.

Relaciones estratigráficas: Sobreyace disconformemente al Miembro Loma Danta, de la Formación Descartes con un contacto erosivo. Está sobreyacida concordantemente por los sedimentos del Miembro Zapotal de la Formación Descartes, el contacto es transicional y está definido por una zona de intercalación de calcilutitas con areniscas conglomerádicas (Denyer, 1977).

Edad geológica: Rivier (1983) le asigna una edad entre el Paleoceno Medio a Superior.
Aspectos específicos: Rivier (1983) indica que debido a la presencia de mantos, bloques y litoclastos andesiticos se podría imaginar un aparato (o fractura) volcánico muy cercano. Astorga (1987) interpreta que esta unidad presenta un origen turbidítico, correspondiente con facies de depósitos de flujos turbulentos gravosos y depósitos de flujos de escombros.

Antecedentes: Fue descrita como unidad informal Cerco de Piedra en las Campañas geológicas de 1976, 1977 y 1978. Sprechman (1982) la describe por primera vez como Formación Conglomerado Cerco de Piedra. Astorga (1987) incluye los sedimentos asociados a esta unidad dentro de la Formación Descartes.

\section{MIEMBRO ZAPOTAL}

Esta compuesta por sedimentos volcaniclásticos de origen turbidíticos del Paleoceno Superior y el Eoceno Inferior aflorantes en el área este del bajo Tempisque (Flores et al., 2003).

\section{GRUPO AGUACATE}

No se detallará esta unidad, pues solo se diferenció su contacto en el mapa geológico. Constituye la cobertura volcánica del final del Mioceno que se pudo extender al Plioceno Inferior. En la hoja Abangares afloran lavas andesíticas, ignimbritas y sedimentos lacustres intercalados. Esta unidad sobreyace disconformemente toda la secuencia volcano-sedimentaria del Crétacico Superior al Eoceno Inferior, anteriormente descrita

\section{AGRADECIMIENTOS}

El presente estudio presenta resultados obtenidos como parte de los proyectos de investigación 113-90-071: "Análisis Geoestructural comparado de Costa Rica" y 808-99-237: Arrecifes fósiles de Costa Rica". 


\section{REFERENCIAS}

AGUILAR, T., 1977: Geología del área de las lomas Monte Potrero y alrededores, cantón de Abangares, provincia de Guanacaste, Costa Rica. - 21 págs. Univ. de Costa Rica, San José. [Inf. Campaña Geol.].

AGUILAR, T. \& DENYER, P., 2001: Una nueva especie de Euphyllia (Scleractinia: caryophylliidae) en las calizas de Barra Honda (Paleógeno), Costa Rica. - Rev. Biol. Trop. Supl. 2:195-201.

AGUILAR, T. \& DENYER, P., 2002: Primer hallazgo de trigonias (Mollusca: bivalvia) cretácicas de Costa Rica (Tortugal, Guanacaste): Geología, estratigrafía y paleontología. - Rev. Geol. Amér. Central, 26: 53-63.

ALVARADO, G.E. \& DENYER, P., 1997: Implications of the Caribbean region of the high-Mg volcanic rocks in the Costa Rican ophiolitic complexes: The case of the Tortugal komatiitic-like suite. - Zbl. Geol. Paläont. 1997(3-6): 409-429.

ALVARADO, G.E., DENYER, P., SINTON, C.W., 1997: A 89 Ma komatiitic suite, Costa Rica: Implications for a common geologic origin of the Caribbean and eastern Pacific region from a mantle plume. - Geology, 25: 439-442.

ASTORGA, A., 1987: El Cretácico Superior y el paleógeno de la vertiente pacífica de Nicaragua meridional y Costa Rica septentrional: Origen, evolución y dinámica de las cuencas profundas relacionadas al margen convergente de Centroamérica. - 115 págs. Univ. de Costa Rica [Tesis Lic.]

CALERO, A., 1987: Geología del cerro Hortigal y alrededores, provincia de Puntarenas, Costa Rica. - 50 págs. Univ. de Costa Rica, San José. [Inf. Campaña Geol.]

CALVO, C., 1987: Las Calizas neríticas de la vertiente pacífica del istmo centroamericano meridional. - 90 págs. Univ. Costa Rica, San José [Tesis Lic.]

CALVO, C. \& BOLZ, A., 1991: La Formación Espíritu Santo (Costa Rica): sistema de plataforma carbonatada autóctona del Paleoceno sup.- Eoceno inf. - Rev. Geol. América Central, 13: 91-95.

CALVO, C., 1998: Kretazische subduktionsprozesse in südzentralamerika. - Profil 15: 1-161.

DENGO, G., 1962: Estudio geológico de la región de Guanacaste, Costa Rica. - 112 págs. Inst. Geogr. Nac. San José.

DENYER, P., 1977: Geología del cerro Zapote y alrededores, Colorado de Abangares, Guanacaste, Costa Rica. - 10 págs. Univ. de Costa Rica. [Inf. Campaña Geológica].
DENYER, P., MONTERO, W., SOTO, G., QUEZADA, A., LEANDRO, L., PÉREZ, C. \& RODRÍGUEZ, D., 1987: Geología y tectónica de la margen oriental del golfo de Nicoya, Costa Rica. - Ciencia y Tecnología 11(2): 17-31.

FLORES, K., 2003: Propuesta tectonoestratigráfica de la región septentrional del golfo de Nicoya, Costa Rica. 176 págs. Univ. de Costa Rica, San José. [Tesis Lic.]

FLORES, K., DENYER, P. \& AGUILAR, T., 2003: Nueva propuesta estratigráfica: geología de las hojas Matambú y Talolinga, Guanacaste, Costa Rica. - Rev. Geol. América Central, 28: 131-138.

GALLI-OLLIVIER, C., 1979: Ophiolite and island arc volcanism in Costa Rica. - Geol. Soc. Amer. Bull. 90: 444-452.

GALLI-OLIVIER, C. \& SCHMITD-EFFING, R, 1974: Estratigrafía de la cubierta sedimentaria supra-ofiolítica Cretácica de Costa Rica. - Cienc. Tec. UCR 1: 87-96.

INTERNATIONAL COMMISSION ON STRATIGRAPHY, 2004: International stratigraphic chart.-ICS. <http://www.stratigraphy.org /chus/pdf> [consulta 7 jun. 2004]

JACCARD, S. \& MUNSTER, M., 2001: Etude géologique multidisciplinaire de la plateforme de Barra Honda (Guanacaste, Costa Rica): sédimentologie, isotopes stables du strontium, du carbone, de Oxygéne et contexte géodynamique- Univ. du Lausanne, Lausanne. [Tesis Diplôme]

JACCARD, S., MUNSTER, M., BAUMGARTNER, P.O., BAUMGARTNER-MORA, C. \& DENYER, P., 2001: Barra Honda (Upper Paleocene - Lower Eocene) and El Viejo (Campanian - Maastrrichtian) carbonate platformas in the Tempisque area (Guanacaste, Costa Rica). - Rev. Geol. Amér. Central, 24: 9-28.

KUIJPERS, E., 1980: The geologic history of the Nicoya Ophiolite Complex, Costa Rica, and its geotectonic significance. - Tectonophysics, 68: 233-255.

MACDONAL, D.F., 1920: Informe final geológico y geográfico de Costa Rica. - Rev. de Costa Rica 2(1):28-32; (2):50-57; (4):106-111; (5):123-125, 139-148.

MORA, S., 1981: Barra Honda. - 94 págs. Ed. UNED, San José.

RIVIER, F., 1983: Síntesis geológica y mapa geológico del área de bajo Tempisque, Guanacaste, Costa Rica. Inf. Sem. IGN, 1983(1):7-30.

ROMANES, J. , 1912: Geological notes on the península de Nicoya, Costa Rica. - Geol. Mag. 9: 258-265. 
SCHMIDT-EFFING, R., 1974: El primer hallazgo de amonites en América Central Meridional y notas sobre facies cretácicas en dicha región. - Inf. Sem. I.G.N. 1975(1): 53-61.

SPRECHMANN, P., 1982: Estratigrafía de Costa Rica (América Central), I: unidades estratigráficas sedimenta- rias. - Actas V Cong. Latinoamericano Geol. Buenos Aires, 1: 55-71.

TOURNON, J., 1984: Magmatismes des Mesozoique a l'actuel en Amérique Centrale: L'exemple de Costa Rica, des ophiolites aux andesites. - 355 págs. Univ. Curie, Paris [Tesis PhD] 


\title{
Fe de errata
}

\section{PEVALUACIÓN DE LA AMENAZA Y CAUSAS DE INUNDACIÓN EN LA CIUDAD DE TURRIALBA, COSTA RICA}

\author{
Elena Badilla
}

Escuela Centroamericana de Geología

email: ebadilla@geologia.ucr.ac.cr

En el artículo publicado en la Revista Geológica de América Central 28: 91-108, la leyenda del mapa de amenaza de inundación (Fig. 11) presenta un error. Se muestra a continuación la versión corregida de este mapa y se aclara que la versión anterior es errónea e inválida.

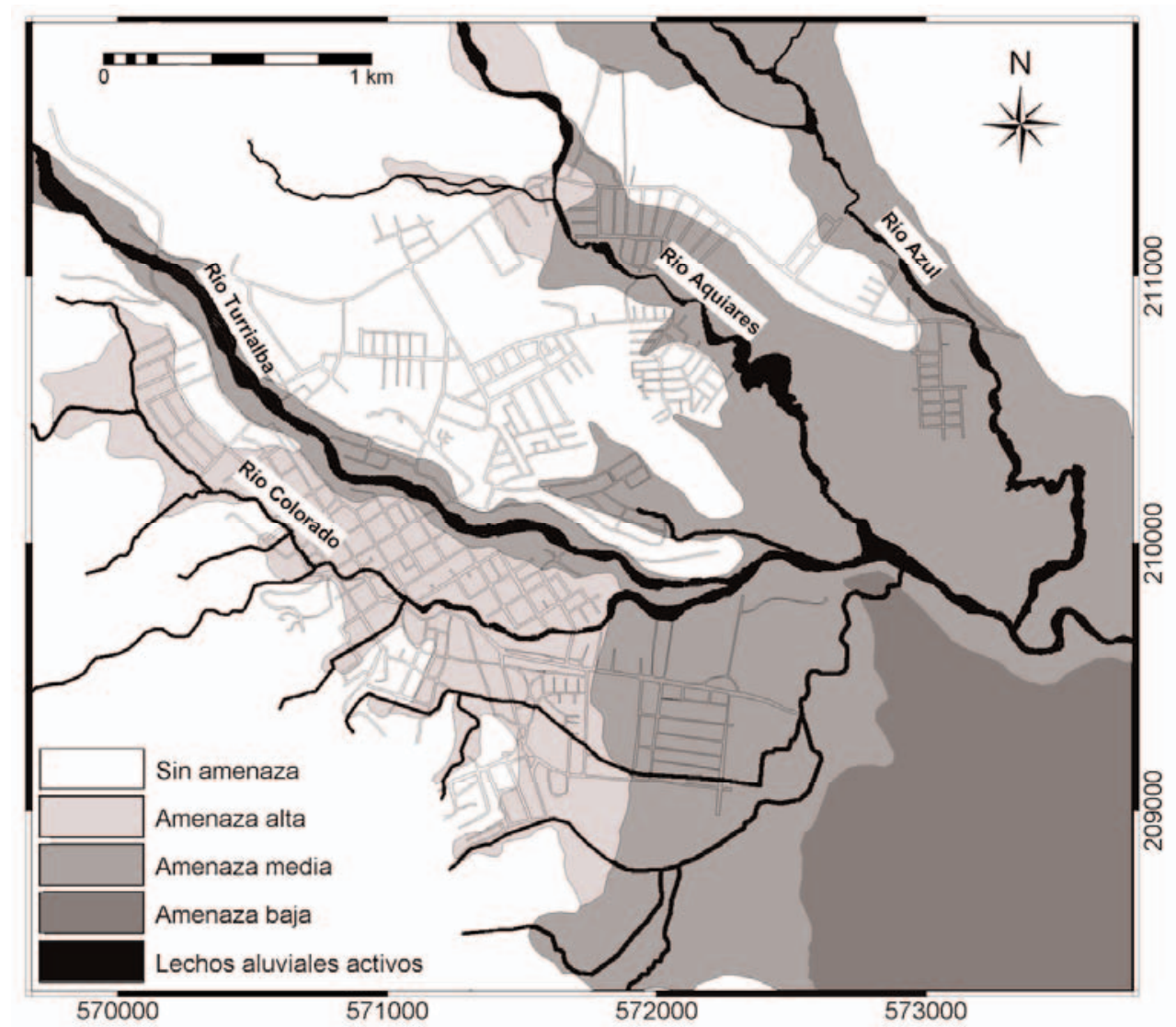

Fig. 11: Mapa de amenaza de inundación del área de estudio. 
